\title{
A K-Means based Model towards Ebola Virus Prorogation Prediction
}

\author{
Baohua LIU \\ Department of Computer \\ Science, Shandong University \\ of Science and Technology, \\ Qingdao 266590, China
}

\author{
Xudong Wang \\ Department of Information \\ System, Shanghai University, \\ Shanghai 201800, China \\ Cong LIU \\ Department of Computer \\ Science, Shandong University \\ of Science and Technology, \\ Qingdao 266590, China
}

\author{
Qi Gao \\ Department of Mathematics, \\ Shandong Normal University, \\ Jinan 250002 China.
}

\begin{abstract}
Ebola hemorrhagic fever is a disease caused by one of five different Ebola viruses. Four of the strains can cause severe illness in humans and animals. Humans can be infected by other humans if they come in contact with body fluids from an infected person or contaminated objects from infected persons. Humans can also be exposed to the virus, for example, by butchering infected animals. Deadly human Ebola outbreaks have been confirmed in the following countries: Democratic Republic of the Congo (DRC), Gabon, South Sudan, Ivory Coast, Uganda, and Republic of the Congo (ROC), Guinea and Liberia. In this sense, it is of vital importance to analysis the history data and predicts its propagation. More specifically, a model based k-means algorithm to determine the optimal locations of virus delivery is constructed and tested Using Mab-lab programming. By experiment, we find that our model can work well and lead to a relatively accurate prediction, which can help the government forecast the epidemic spread more efficiently.
\end{abstract}

Keywords: Date Mining; K-Means Technique; Algorithm Complexity; Ebola Infect Prediction; Mat-lab Simulate

\section{INTRODUCTION}

Ebola also known Ebola virus, which is a very rare virus in southern Sudan in 1976 and Izard Ebola River discovery of its existence, hence the name ${ }^{[1]}$. According to the World Health Organization's message, since December 2013, the outbreak of the Ebola virus outbreak in West Africa continued, in Guinea, Sierra Leone and Liberia infected at least 567 people, including 350 death $^{[2]}$. The disease has a high risk of death, killing between 25 and 90 percent of those infected with an average of about 50 percent and has caused immense sorrow especially for the African people ${ }^{[3]}$.If there is no effective drug control measures and the epidemic will continue to spread it, and spread to the whole world.

Based on the data from World Health Organization, Ebola virus does not spread through the air, and no evidence has proved that the virus undergo the variation. Mode of transmission of Ebola virus is close direct contact with body fluids of patients, including the patient's blood, excrement, vomit which serve the strongest infection. Virus can also be found in saliva and tears.

We build a model to simulate the spreading of the disease, a model based k-means algorithm to determine the optimal locations of delivery. By the experiment, we find our model can work well, they can help government forecast the epidemic spread, and can save a lot of resource.

\section{FORECASTING OF EBOLA CRISIS}

Ebola outbreak in Guinea after a steady period of time and, more recently was "looked up" trend. 2014, ravaged West African country of Guinea, Liberia, Sierra Leone, the Ebola virus spreading at an alarming rate. By February 6, 2015 $5^{[4]}$, the World Health Organization had reported 22,525 confirmed, probable and suspected cases in West Africa, with 2988 in
Guinea, 8745 in Liberia and 10792 in Sierra Leone. What is worth, it has caused 9004 deaths.

Ebola outbreak in Guinea after a steady period of time and, more recently was "looked up" trend. We care about the few infected person can infect assigned to the crowd, with the passage of time, whether the disease will spread, causing many people are infected. It cannot wait to predict the ratio of Ebola virus spreads.

As it is shown in table 1, we have found some data from the site of WHO (World Health Organization). By these data, we can discover the tread of Ebola virus spreads.

Table 1. number of infection in Guinea, Liberia, Sierra Leone

\begin{tabular}{|c|c|c|c|}
\hline & Guinea & Liberia & Sierra Leone \\
\hline $2014-11-24$ & & 2753 & \\
\hline $2014-11-25$ & 1892 & & 5595 \\
\hline $2014-11-28$ & 1921 & 2801 & 5831 \\
\hline $2014-11-29$ & & 2805 & \\
\hline $2014-11-30$ & 1929 & & 5978 \\
\hline $2014-12-1$ & & & 6039 \\
\hline $2014-12-2$ & 1949 & 2824 & 6201 \\
\hline $2014-12-3$ & 1956 & 2830 & \\
\hline $2014-12-6$ & 2035 & & 6317 \\
\hline $2014-12-7$ & 2051 & 2869 & 6375 \\
\hline $2014-12-9$ & 2081 & 2946 & 6457 \\
\hline $2014-12-10$ & 2096 & & 6497 \\
\hline $2014-12-13$ & 2115 & & 6638 \\
\hline $2014-12-14$ & 2127 & 3021 & 6702 \\
\hline $2014-12-16$ & 2164 & & \\
\hline $2014-12-17$ & & & 6856 \\
\hline $2014-12-18$ & & 3085 & \\
\hline
\end{tabular}




\begin{tabular}{|c|c|c|c|}
\hline $2014-12-20$ & 2259 & 3085 & 6975 \\
\hline $2014-12-21$ & 2284 & & 7017 \\
\hline $2014-12-24$ & 2342 & 3108 & 7160 \\
\hline $2014-12-27$ & 2384 & & 7326 \\
\hline $2014-12-28$ & 2397 & 3110 & 7354 \\
\hline $2014-12-31$ & 2435 & 3116 & 7476 \\
\hline $2015-1-2$ & & 3118 & \\
\hline $2015-1-3$ & 2465 & 3118 & 7570 \\
\hline $2015-1-4$ & 2471 & & 7602 \\
\hline $2015-1-5$ & 2477 & & \\
\hline $2015-1-6$ & & 3123 & 7759 \\
\hline $2015-1-7$ & 2493 & 3123 & \\
\hline $2015-1-10$ & 2508 & & 7766 \\
\hline $2015-1-11$ & 2514 & 3127 & 7786 \\
\hline $2015-1-12$ & 2522 & 3127 & 7802 \\
\hline $2015-1-13$ & 2525 & & 7825 \\
\hline $2015-1-15$ & & 3131 & \\
\hline $2015-1-17$ & 2539 & & 7883 \\
\hline $2015-1-18$ & 2539 & 3135 & 7903 \\
\hline $2015-1-19$ & 2542 & & 7909 \\
\hline $2015-1-20$ & 2545 & 3136 & 7921 \\
\hline $2015-1-24$ & 2559 & & 7963 \\
\hline $2015-1-25$ & 2569 & 3138 & 7968 \\
\hline $2015-1-26$ & 2571 & 3138 & 7977 \\
\hline $2015-1-27$ & 2575 & & 7989 \\
\hline $2015-1-28$ & & 3138 & \\
\hline $2015-1-31$ & 2593 & 3143 & 8042 \\
\hline $2015-2-1$ & 2608 & 3143 & 8059 \\
\hline $2015-2-2$ & 2621 & & 8063 \\
\hline $2015-2-3$ & 2628 & & 8084 \\
\hline
\end{tabular}

\subsection{Ebola virus infection forecast model without drug}

To make it easier to understand the model, we first list the symbols used in the following table.

Table 2. Symbols used in the model

\begin{tabular}{c|c}
\hline $\mathrm{i}(\mathrm{t})$ & $\begin{array}{c}\text { the number of infected } \\
\text { individuals in the } \mathrm{t} \text { day } \\
\text { the number of susceptible } \\
\mathrm{s}(\mathrm{t})\end{array}$ \\
$\mathrm{individuals}$ in the $\mathrm{t}$ day \\
$\mathrm{N}$ & total people in the country \\
$\mathrm{k}$ & infection coefficient \\
\hline
\end{tabular}

\section{Assumption}

(1) $s(t)$ represents the number of susceptible people in the t day, and $i(t)$ represents the number of infection people in the $t$ day.

(2) Each day it increase $\mathrm{k} 0$ infection, and $\mathrm{k}\urcorner 0$ can be calculated by the following equation: $\mathrm{K} 0=\mathrm{k} * \mathrm{~s}(\mathrm{t})$.

(3) People will not die in the country, that is to say, $s(t)+i(t)=N$.

By the three assumptions, we can obtain the following differential equation[7-9]:

Solve the differential equation, we achieve

Figure 1 shows the trend of $\mathrm{i}(\mathrm{t})$ with the increasing of $\mathrm{t}$.

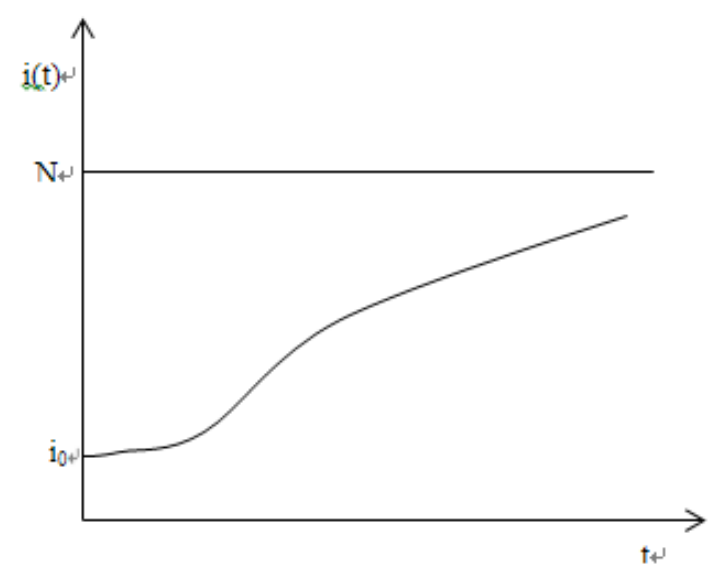

Figure 1. Change of the infected people

We can see from the chart, the number of infection people is increasing with $t$, the speed is very quick at the start time, but when there are a lot of people infected, the speed is become low. When $\mathrm{t} \rightarrow \infty, \mathrm{i}(\mathrm{t}) \rightarrow \mathrm{N}$.

According to the model, and combine with the data in table 1 ,we obtain the infection trend in Sierra Leone by matlab ${ }^{[5]}$, it is showed in Figure 2.

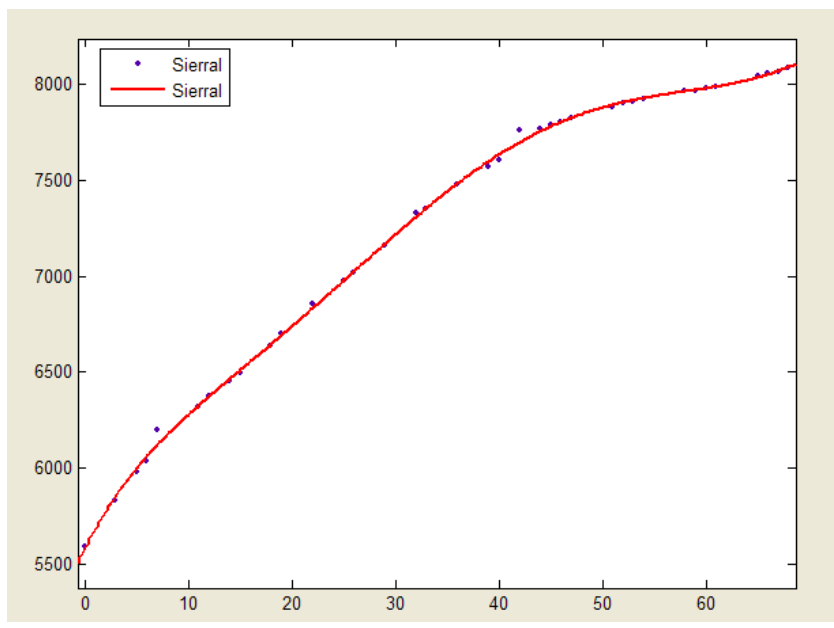

Figure 2. Trend of number of infected people in Sierra Leone

We select November 25, 2014 as the first day, use matlab plot the chart, it can be seen from the chart that the infection ratio is decline. We predict the future infection number by the model, list them in table 3.

Table 3 Number of the infected in the next five days

\begin{tabular}{|c|c|c|c|c|c|}
\hline time & $2-4$ & $2-5$ & $2-6$ & $2-7$ & $2-8$ \\
\hline number & 8089 & 8116 & 8146 & 8180 & 8219 \\
\hline
\end{tabular}

\subsection{The SIR model}

Assume the world medical association has developed a new medication that can stop Ebola and cure patients whose disease is not advanced, the model above can not meet the situation, so we build the following model. As the first step in the modeling process, we identify the independent and dependent variables. The independent variable is time $t$, measured in days. 
To make it easier to understand the model, we first list the symbols used in the following table.

Table 4. Symbols used in the model

\begin{tabular}{|c|c|}
\hline $\begin{array}{c}\mathrm{S}(\mathrm{t}) \\
\mathrm{I}(\mathrm{t}) \\
\mathrm{R}(\mathrm{t}) \\
\mathrm{N} \\
\mathrm{s}(\mathrm{t})=\mathrm{S}(\mathrm{t}) / \mathrm{N} \\
\mathrm{i}(\mathrm{t})=\mathrm{I}(\mathrm{t}) / \mathrm{N} \\
\mathrm{r}(\mathrm{t})=\mathrm{R}(\mathrm{t}) / \mathrm{N} \\
\mathrm{b} \\
\mathrm{k}\end{array}$ & $\begin{array}{c}\text { the number of susceptible } \\
\text { individuals } \\
\text { the number of infected } \\
\text { individuals } \\
\text { the number of recovered } \\
\text { individuals } \\
\text { the total population in the } \\
\text { country } \\
\text { the susceptible fraction of the } \\
\text { population } \\
\text { the infected fraction of the } \\
\text { population } \\
\text { the recovered fraction of the } \\
\text { population } \\
\text { the number of people } \\
\text { infested contact } \\
\text { recover ratio }\end{array}$ \\
\hline
\end{tabular}

\section{Assumption}

(1) No one is added to the susceptible group, since we are ignoring births and immigration. The only way an individual leaves the susceptible group is by becoming infected. We assume that the time-rate of change of $S(t)$, the number of susceptibles, 1 depends on the number already susceptible, the number of individuals already infected, and the amount of contact between susceptible and infected. In particular, suppose that each infected individual has a fixed number $b$ of contacts per day that are sufficient to spread the disease. Not all these contacts are with susceptible individuals. If we assume a homogeneous mixing of the population, the fraction of these contacts that are with susceptible is $\mathrm{s}(\mathrm{t})$. Thus, on average, each infected individual generates $\mathrm{b} s(\mathrm{t})$ new infected individuals per day. With a large susceptible population and a relatively small infected population, we can ignore tricky counting situations such as a single susceptible encountering more than one infected in a given day.

(2) We also assume that a fixed fraction $\mathrm{k}$ of the infected group will recover during any given day. For example, if the average duration of infection is three days, then, on average, one-third of the currently infected population recovers each day. (Strictly speaking, what we mean by "infected" is really "infectious," that is, capable of spreading the disease to a susceptible person. A "recovered" person can still feel miserable, and might even die later from pneumonia.)

It may seem more natural to work with population counts, but some of our calculations will be simpler if we use the fractions instead. We complete our model by giving each differential equation an initial condition. Our complete model is:

\section{(3)}

We don't know values for the parameters $\mathrm{b}$ and $\mathrm{k}$ yet, but we can estimate them according to the data. We can use matlab to solve the differential equation and use is to plot the chart.

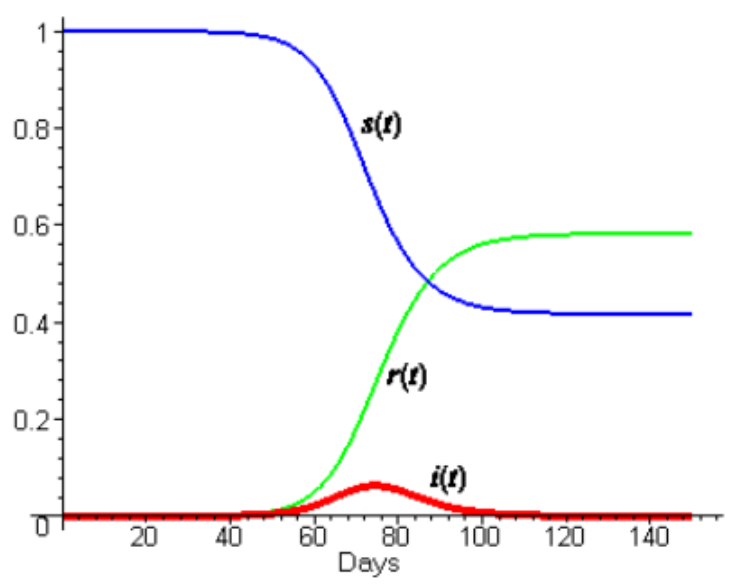

Figure 3. Change of ratios of infected, susceptible and removed people

From the chart, we can see before drugs play a part, the number of infection is increasing with time, achieve a peak value, then the number of infection will decrease by zero. After treatment, the number of susceptible plus the number of recover and death is the number of people in the country.

\section{DETERMINING THE LOCATIONS OF DELIVERY}

For a simpler demonstration of our model, we take the country of Sierra Leone to be the scope of consideration. The official site of the WHO provided two maps showing respectively the situation of transmission of the disease and the information regarding the currently opened Ebola treatment centers. For a more informative view, we combined the two maps:

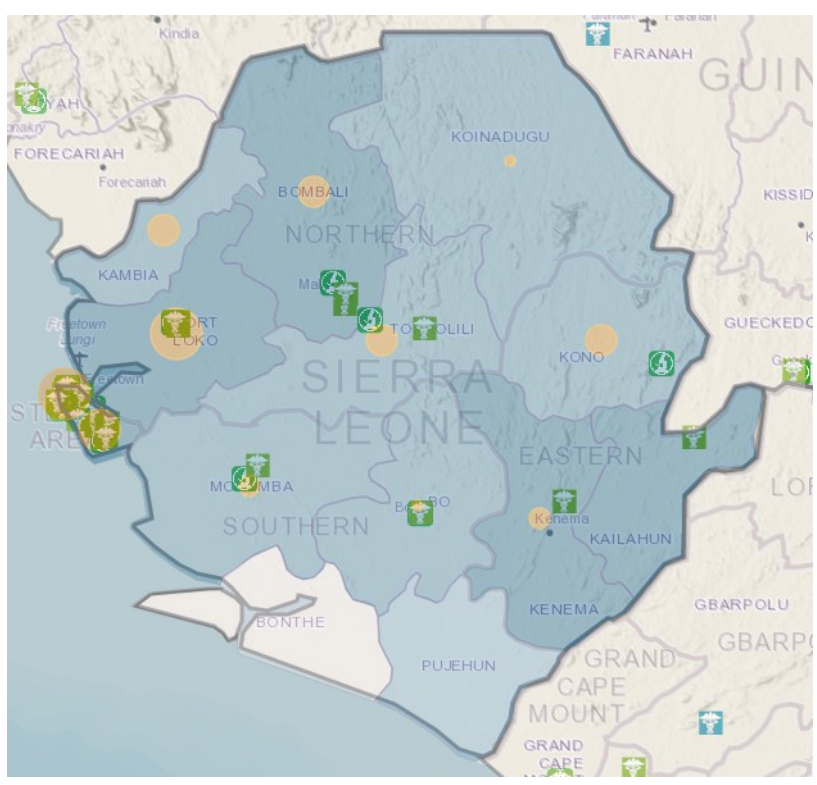

Figure 4. Transmission of Ebola in Sierra Leone

Symbols like this reveal that the locations of the currently functioning Ebola treatment centers. And the coloring of the regions reflects how heavily it has been hit by the disease in the long term. The heavier the color, the larger is the number of accumulated cases of infection. Finally the orange circles show how well the spreading of the disease has been contained in 
those particular regions. Larger circles mean that more cases of infection have been confirmed over the last 21 days.

We suppose that multiple locations of initial deliveries rather than only one location of initial delivery will later result in easier and quicker distribution of the medication. In this particular case, we assume that initial deliveries will be made to three locations within the country.

In order to determine the locations for greater efficiency, we consider the locations of the Ebola treatment centers. We try to divide the treatment centers into three groups in such a way that treatment centers within the same group are geographically relatively closer to each other while the geographical distances are relatively larger between treatment centers that belong to different groups. We then determine one location of delivery within each of the three groups. For the purpose of the grouping of the treatment centers, we adopt the k-means clustering algorithm ${ }^{[6]}$

First of all, we establish a certain rectangular coordinate system on the map and determine accordingly the coordinates of each of the Ebola treatment centers as shown in the figure below:

In the west-most part of the country where there are quite a number of treatment centers located very near to each other, we pick only one of them to be considered in the clustering process.

The geographical distances as the crow flies between any two of the Ebola treatment centers are listed in the matrix below:

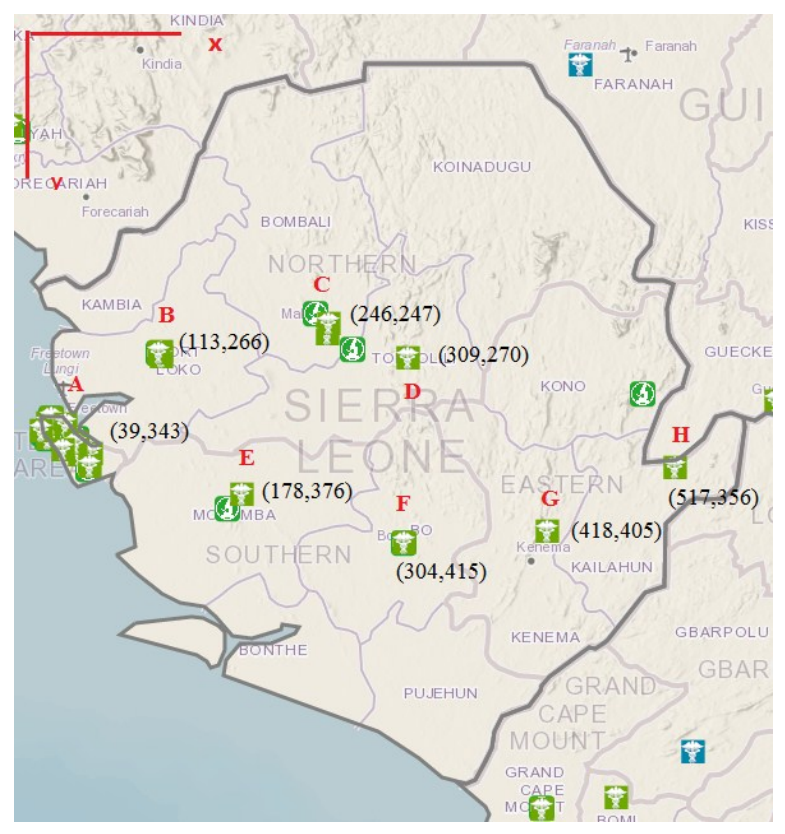

Figure 5. Geographical locations of ETCs in Sierra Leone

Table 5. Distances between ETCs

\begin{tabular}{|c|c|c|c|c|c|c|c|c|}
\hline & $\mathrm{A}$ & $\mathrm{B}$ & $\mathrm{C}$ & $\mathrm{D}$ & $\mathrm{E}$ & $\mathrm{F}$ & $\mathrm{G}$ & $\mathrm{H}$ \\
\hline \multirow{2}{*}{$\mathrm{A}$} & 0 & 106. & 228. & 279. & 142. & 274. & 384. & 478. \\
& & 794 & 178 & 694 & 864 & 607 & 038 & 177 \\
\hline \multirow{2}{*}{$\mathrm{B}$} & 106. & \multirow{2}{*}{0} & 134. & 196. & 127. & 242. & 335. & 413. \\
& 794 & & 35 & 041 & 769 & 244 & 181 & 903 \\
\hline \multirow{2}{*}{$\mathrm{C}$} & 228. & 134. & 0 & 67.0 & 145. & 177. & 233. & 292. \\
& 178 & 35 & & 671 & 825 & 73 & 555 & 099 \\
\hline \multirow{2}{*}{$\mathrm{D}$} & 279. & 196. & 67.0 & \multirow{2}{*}{0} & 168. & 145. & 173. & 225. \\
& 694 & 041 & 671 & & 514 & 086 & 511 & 078 \\
\hline
\end{tabular}

\begin{tabular}{|c|c|c|c|c|c|c|c|c|}
\hline \multirow{2}{*}{$\mathrm{E}$} & 142. & 127. & 145. & 168. & 0 & 131. & 241. & 339. \\
& 864 & 769 & 825 & 514 & & 898 & 746 & 589 \\
\hline \multirow{2}{*}{$\mathrm{F}$} & 274. & 242. & 177. & 145. & 131. & 0 & 114. & 221. \\
& 607 & 244 & 73 & 086 & 898 & 0 & 438 & 02 \\
\hline \multirow{2}{*}{$\mathrm{G}$} & 384. & 335. & 233. & 173. & 241. & 114. & 0 & 110. \\
& 038 & 181 & 555 & 511 & 746 & 438 & & 463 \\
\hline \multirow{2}{*}{$\mathrm{H}$} & 478. & 413. & 292. & 225. & 339. & 221. & 110. & 0 \\
& 177 & 903 & 099 & 078 & 589 & 02 & 463 & 0 \\
\hline
\end{tabular}

\section{Algorithm:}

First we choose three of the initial centers of the three clusters denoted as: Cluster $_{i}(\mathrm{i}=1,2,3)$.

We then consider the distances between each of the rest of the treatment centers and each of the three treatment centers chosen as the center of the clusters $D_{\text {p,ci. }}$.

Where $p$ is any one of the rest of the treatment centers and $c_{i}$ is any of the centers of the clusters, namely, at this particular moment, the treatment centers chosen as the initial centers of the clusters.

If for a given $\mathrm{p}, \min \left\{\mathrm{D}_{\mathrm{p}, \mathrm{c} i}\right\}=\mathrm{D}_{\mathrm{p}, \mathrm{c} j}$, then $\mathrm{p}$ belongs to the $\mathrm{jth}$ cluster.

In the next iteration we first compute the new centers of Cluster $(i=1,2,3)$ as the means of $p_{i}$, where $p_{i}$ is the treatment centers already assigned to Cluster $\mathrm{i}$ :

Where $n_{i}$ is the number of treatment centers in Cluster $i, x_{i, j}$ is the $\mathrm{x}$ coordinate of the $\mathrm{jth}$ treatment center in Cluster ${ }_{i}$ and $\mathrm{y}_{\mathrm{i}, \mathrm{j}}$ is the $y$ coordinate of the jth treatment center in Cluster ${ }_{i}$.

We compute again $\min \left\{\mathrm{D}_{\mathrm{p}, \mathrm{ci}}\right\}$ for every $\mathrm{i}$ and determine once more time which cluster each treatment center belongs to in light of the new $\mathrm{c}_{\mathrm{i}}$ for each Cluster $\mathrm{i}_{\text {. }}$

We repeat this process until not a single one treatment center transmits from one cluster to another within an iteration.

At this time we take the clusters as the final result.

\section{Solution:}

First we take $\mathrm{A}=(39,343), \mathrm{C}=(246,247), \mathrm{H}=(517,356)$ as the initial centers for each of the three clusters, see in figure 7.

And we apply the algorithm to the map, achieve the result shown in figure 8 .

The result of the second iteration is exactly the same as the first. And the final result is depicted in the following figure 9.

Then we take the centers of each of the clusters as the initial delivery locations: $\mathrm{c}_{1}=(110,328), \mathrm{c}_{2}=(286,311), \mathrm{c}_{3}=(468$, 380).

After the initial deliveries are made, mediation should be then transmitted to the Ebola treatment centers within the corresponding cluster where they can be stored, organized and utilized for the treatment of the Ebola disease. 


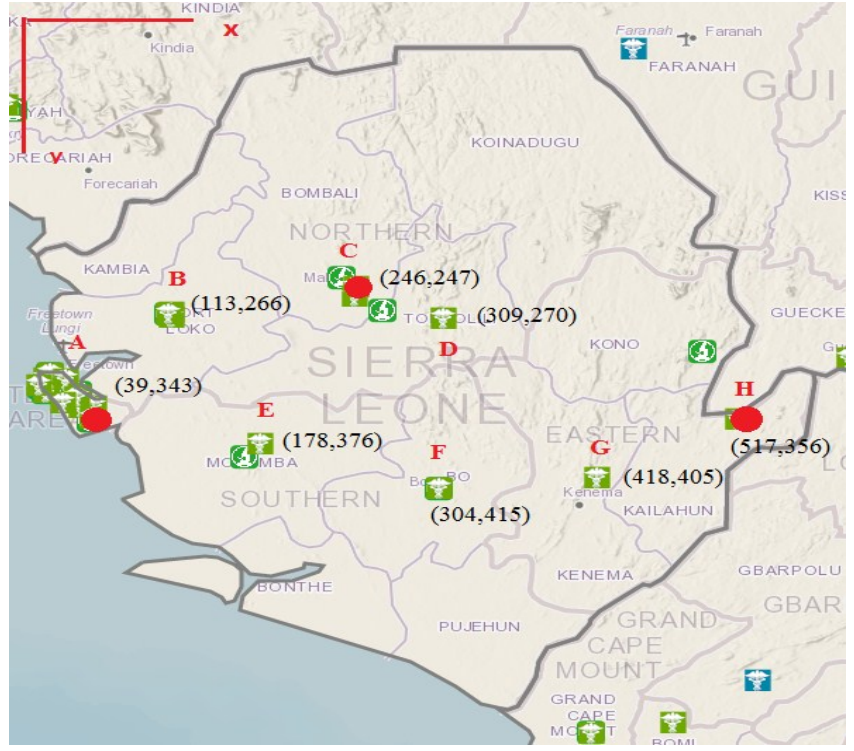

Figure 7. The initial centers of the clusters

\section{CONCLUSION}

When modeling the propagation of the disease, we not only adopt a fitting algorithm based on the relevant data obtained from the WHO official website, but we also discuss the incorporation of the SIR model which takes into account the dynamics of an infectious disease itself.

To help plan out the delivery of the medication, we not only consider the transmission of the disease but we also take into account the locations of the Ebola treatment centers currently established. This adds to the practicality of our model.

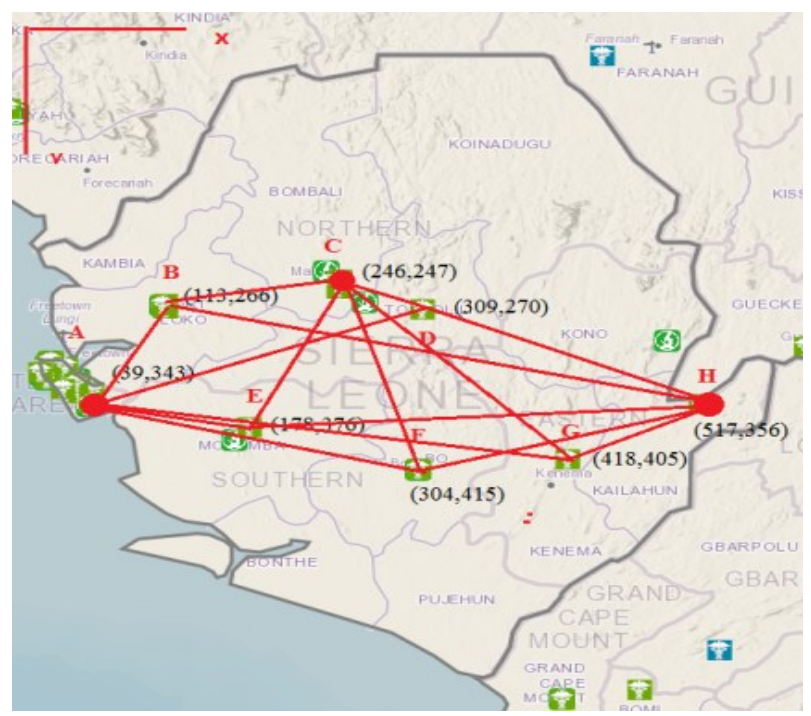

Figure 8 . The crow-fly distances of ETCs

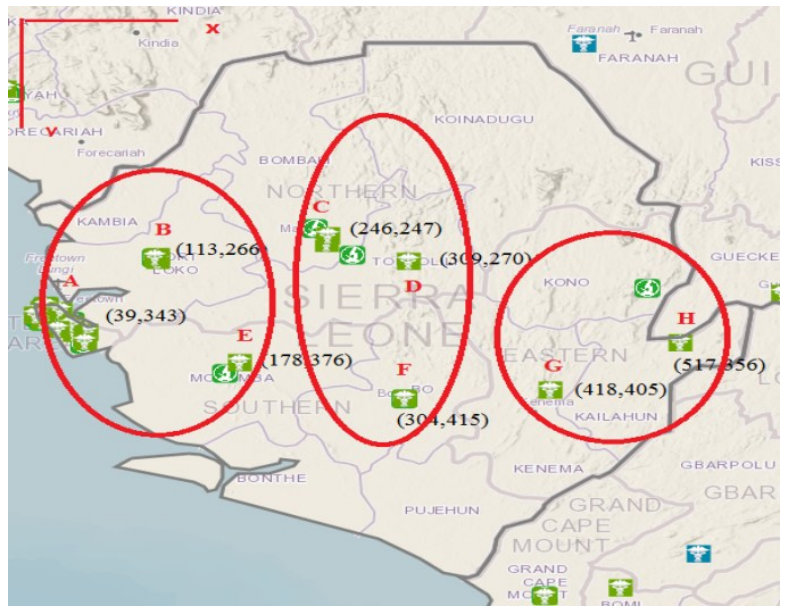

Figure 9 Result of the clustering algorithm

\section{ACKNOWLEDGMENTS}

This work was supported in part by the Graduate Innovation Foundation Project of Shan-dong University of Science and Technology (YC140106). Also, we would like to thank those anonymous reviewers for their constructive comments to help us improve the paper.

\section{REFERENCES}

[1] L. Zhang, H. Wang. Forty years of the war against Ebola. Zhejiang Univ-Sci B 2014; 15(9): 761-765.

[2] "Ebola virus disease Fact sheet No. 103". World Health Organization. September 2014.

[3] Birmingham, K and Cooney, S. Ebola: small, but real progress (news feature). Nature Med 2002;8:313.

[4] http://apps.who.int/gho/data/view.ebola-sitrep.ebolasummary-20150206?lang=en

[5] Zhuo Jinwu. Matlab in the application of mathematical modeling. Beijing university of aeronautics and astronautics. 2011.

[6] Jiawei Han. Data Mining. China Machine Press. 2013. 